Short Communication

\title{
Therapeutic Musical Scales: Theory and Practice
}

Riccardo Misto *

Villaggio S. Antonio onlus via Cappello 79, Noventa Padovana (Padova), Italy; E-Mail: ricmisto@gmail.com

* Correspondence: Riccardo Misto; E-Mail: ricmisto@gmail.com

Academic Editor: Peter Fraenkel

Special Issue: Integrating Music and Other Arts in Therapy

OBM Integrative and Complementary Medicine 2021, volume 6 , issue 2

doi:10.21926/obm.icm.2102019
Received: November 19,2020

Accepted: April 17,2021

Published: May 24,2021

\begin{abstract}
Using musical scales in a therapeutic key is one of the fundamental music therapy techniques of the Yoga of Sound (Nāda Yoga). The practice consists of singing particular sound formulas (scales), which are devised on a logical mathematical basis formed by specific musical intervals. These scales can bring to the surface, in a clear (objective), recognizable, and predictable way, psycho-emotional states and transform the blocked emotional energies. These blocked emotional energies are caused by repeated emotional stress, which, according to the psychosomatic principle, is the main cause of the physical and mental problems and pathologies. In this article, the fundamental principles of this music therapy, in theory and practice, are uncovered and analyzed.
\end{abstract}

\section{Keywords}

Music therapy; Nāda Yoga; yoga of sound; musical scales; blocked emotional energies; Indian raga; melakartha scale system

\section{Introduction}

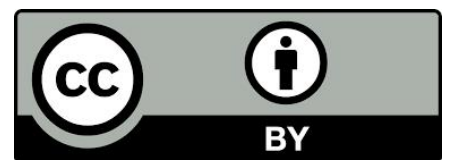

(C) 2021 by the author. This is an open access article distributed under the conditions of the Creative Commons by Attribution License, which permits unrestricted use, distribution, and reproduction in any medium or format, provided the original work is correctly cited. 


\subsection{Methodology and Assumptions}

The proposed procedure involves simple and elementary singing of melodic structures (scales or modes) in both ascending and descending phases. A pre-established particular rhythmic progression that favors the movement of vital energy promotes and enhances the transformative effect, which results in the conversion of blocked emotional energy into positivity in terms of creativity and self-therapy. Particular singing methods, with specific phonemes (sargam), embellishments (gamaka), and derived melodic structures (formations), enhance the overall final effect. It is interesting to note how the singing of musical scales was also in use in the Greek world, especially in the School of Pythagoras. The fact that musical formulas (scales) were already in use in the past for evolutionary and transformative purposes constitutes a historical and cultural element that gives foundation to the theory and practice used today in the Nāda Yoga therapeutic music system.

The technique works on the following levels of the being:

1) Physical-corporeal: The singing vibration is addressed to specific points/areas of the physical body, based on correspondence with the different pitches of the sounds produced and with the phonemes used, which direct the vibration in a vectorial manner. Practical experience indicates how it is possible to determine the specific points or areas of the human body where the vibration is mainly addressed, depending on the type of the phoneme used, its execution mode, and the tone in frequency. Of course, there will also be other reverberation points, depending on the muscle and bone structures of the person producing the sound vibrations.

2) Psycho-emotional: The movement of the notes (intervals), based on the relationship with the tonic note (also considering the influence that each note exerts on the preceding and the following note, increasing or diminishing the basic emotional values), brings out precise emotional states. This is predictable on objective bases, which are found in any subject regardless of nationality, culture, age, sex, and personal history. While singing the sound models, there is a progressive positive transformation in the emotional quality. This criterion is the basis of the classical music system of India (Hindustani and Carnatic).

3) Mental-intellectual: The brain is automatically involved in the processes of calculation, proportion, neuronal connection, and the implementation of cerebral synapses, all of which are elements that contribute to increasing intellectual efficiency (i.e., intelligence). In this context, the scales that are based on a mathematical formula of intervals have the characteristics of being "coherent" (i.e., the same sequence of intervals for both ascending and descending phases) and "specular/symmetrical" (i.e., the first portion of intervals is repeated in the second portion). Such characteristics are quite rare and are found only in some scales. For example, the Dorian mode is equivalent to the scale number 22 of the Melakartha System of South India: Ragam Karaharapriya. 


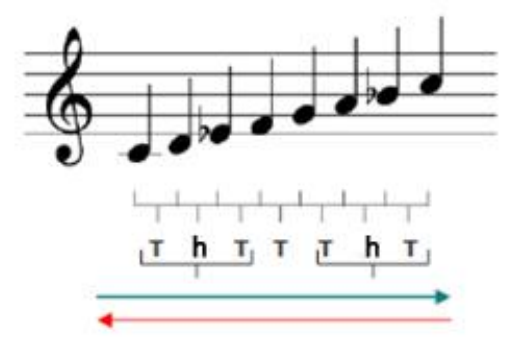

Figure 1 Melakartha System Scale no. 22: Ragam Karaharapriya (Dorian mode) ( $\mathrm{T}=$ tone, $\mathrm{h}=$ half tone).

From this perspective, it is possible to devise particular combinations of scales in order to obtain coherence of the two singing senses (ascending/descending), which is comparable to the phenomenon of what is referred to as a "palindrome" (i.e., reading of a word or phrase in both senses).

The modal scales, which are the bases of the compositions in Indian classical music (Ragas) [1], have the property of being able to arouse precise and objective emotions in the listener. This perception is more prevalent in the singer of the scales/Ragas.

The various scales in the form of a mathematical formula implement a progressive action of the emergence of emotions and their transformation into something else, until the final resolution with the last interval, in which the cathartic process ends.

"The modal development allows the exact perception and immediate classification of every note of the sound elements. The modal system permits, therefore, a much more accurate, powerful, and detailed outlining of the expression." [2]

The following scheme summarizes the main moods associated with the various intervals. For the convenience of our readers, we refer to the key of $C$, but the effect is the same in any key. In any part of the world, the same effect has been found, and this allows us to use musical scales as a therapeutic means to transform emotional energy blocks.

$\begin{array}{cl}\text { TONIC-TONIC } & \text { ASSOCIATED MOODS } \\ \text { C-C } & \text { CALM, LACK OF EMOTION } \\ \text { C-C\# } & \text { ANXIETY, DISCOMFORT, FERMENT, FEAR } \\ \text { C-D } & \text { POSITIVE MOOD, WAIT } \\ \text { C-D\# } & \text { MELANCHOLY, SENTIMENTAL } \\ \text { C-E } & \text { STRENGTH, JOY } \\ \text { C-F } & \text { COMPASSION } \\ \text { C-F\# } & \text { LOVING SADNESS } \\ \text { C-G } & \text { JOY, LOVE, STRENGTH } \\ \text { C-G\# } & \text { ANXIETY, WORRY, SADNESS } \\ \text { C-A } & \text { FORCE, COURAGE } \\ \text { C-A\# } & \text { DELICACY, ATTRACTION, SENSUALITY } \\ \text { C-B } & \text { EVALUATION, CHALLENGE, ANGER }\end{array}$

In the singing practice, it is also important to highlight how breathing is deeply involved, with all the implications concerning the duration of inhalation, holding the breath, and singing (exhalation). 
Recent scientific discoveries [3-5] show that thanks to a regular and proportional movement of the breath, there are positive effects on the vagus nerve, which is responsible for important vital functions concerning the heart, lungs, and stomach. One of the most relevant effects of rhythmic breathing is "cardiac coherence". "With cardiac coherence, or control of heart rate variability, we refer to a therapeutic method and relaxation technique, similar to self-hypnosis and autogenic training, based on breathing control techniques; the technique is aimed at regulating the variability of heart rate and rhythm, and thus influencing the central nervous system through the interaction with the autonomic nervous system, through the vagus nerve (according to the neurovisceral and synchronization model of the parasympathetic and sympathetic systems)" [6].

Finally, the practice of singing therapeutic musical scales is particularly effective when the subject sings on the bases of his/her own "personal tonic note" [7] (i.e., a particular frequency obtainable from one's speaking voice). This corresponds to a psychophysical state of absolute calm (i.e., not opposed to a previous state of agitation). It is based on centering, emotional stability, and maximum physical and mental efficiency, which is physically located at the navel.

\section{Materials and Methods [8-13]}

\subsection{The Method}

Regarding the practice, the singing of therapeutic musical scales is based on a structured formula: singing in the ascending and descending mode and making each note last 4 beats, then 2 beats, 1 beat, half, and half again. In this way, breathing is naturally structured to move the subtle energy of what is referred to as Prana, more consistently and effectively. The emotional and physical effect of vibration is enhanced when the phonemes corresponding to the notes as spelled in the South Indian music system are used:

SA RI (RE in North Indian Hindustani system) GA MA PA DHA NI SA

Each note is to be intended as an interval, with the effective value depending on the tonic.

The specific phonemes have the property of directing the vibratory energy to particular and predictable points or areas of the physical body, while the relationship with the tonic note determines the progressive emergence of precise emotional states, which are gradually transformed until the final conversion with the return on the root (or the tonic note) in the octave.

SA moves toward the navel.

RI produces a strong vibration that descends from the oral cavity area down to the heart.

$G A$ is directed down to the base of the spine at the back of the body.

MA connects the brain area (M) with the heart area (A).

PA goes outward.

DHA is concentrated in the chest, bronchi, and lungs.

$\mathrm{NI}$ reaches the nose-eye band.

We can say that the scales-singing practice always acts on two levels: one physical and the other psycho-emotional. The increase in the speed of singing the sequence provided by the model of each scale contributes to the transformative effect, especially in the scales that bring out very strong emotions, such as anxiety, fear, sadness, and depression. For the definitive transformation of these emotional states, it is recommended to conclude the practice by modifying the heptatonic scale (with 7 different notes) into a pentatonic scale (with 5 notes), in which those 
intervals, which activate the "negative" emotions, are omitted. This does not allow unpleasant emotional residues to remain, despite the previous transformation carried out with the singing of the heptatonic scale.

At the beginning of the practice, it may be useful, especially for those who have difficulty in correctly pronouncing the specific phonemes (sargam), to use a simpler modality (called akar) that consists of singing and always intoning the phoneme A. In this way, among other things, one can better determine the points of the body to which the vibration for each note is mainly directed. The intensity of the emotion(s) evoked by the various intervals can be reinforced with the use of appropriate embellishments (gamakas), among which the vibrato, the wavy, the glissato (or glissando), and the crescendo are particularly important. In addition to repeating the significant intervals in singing to bring out the desired emotional state, further reinforcement is possible by creating specific formations in which the intervals of the scale are combined with particular formulas to enhance their transformative effect.

\subsection{The Four Basic Scales}

In the musical system of South India (Carnatic), the Melakartha System organizes 72 scales, which are divided into 2 sections with 6 sectors each. In each sector, specific emotional states are enhanced with different shades, depending on the mathematical formula applied. As we previously highlighted, the emotional value is clear and objective as the scale is considered and used in a "modal" way, that is, without the use of harmony (chords) but providing only a solid base sound called tonic or drone. In the music therapy practice, we can foresee 4 basic scales, which, according to their mathematical formula, cover all 12 intervals and, therefore, the main emotional types. The following are the scales along with the related emotional moods:

A) DHIRASHANKARABARANAM (number 29 of Melakartha) corresponds to the Major scale or the Ionian mode. Its mood is joyful, serene, and positive. It is used for grounding and emotional stability.

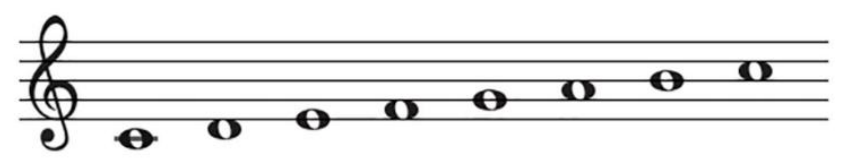

Figure 2 Major scale (Ionian mode) notation

If we analyze the musical intervals ( $C-D, C-E, C-F, C-G, C-A, C-B)$, we can see, by referring to the scheme provided earlier, that they all express good and positive emotions.

B) KARAHARAPRIYA (number 22 of Melakartha) corresponds to the Dorian mode. Its mood is calm, with slight melancholy. It is used to begin by gently removing sadness and then bring calmness and serenity. 


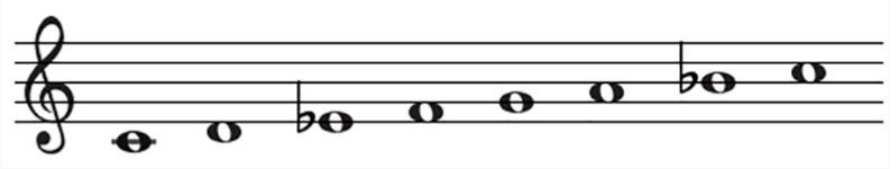

Figure 3 Dorian mode notation

In this scale formula, interval C-Eb expresses light melancholy and interval C-Bb expresses delicacy and sensuality.

C) MLECHAKALIYANI (number 65 of Melakartha) corresponds to the Lydian mode. It touches affective and sentimental suffering in a precise way.

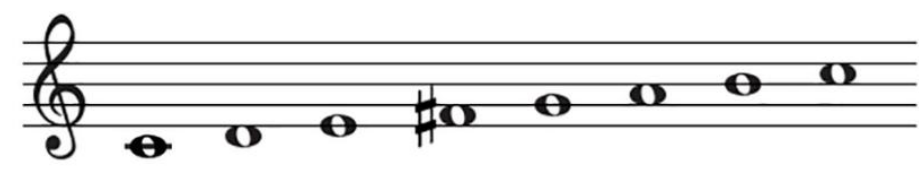

Figure 4 Lydian mode notation

This scale formula has the C-F\# interval, which enhances sentimental sadness.

D) MAYAMALAVAGOWLA (number 15 of Melakartha) corresponds to the Double Harmonic Major scale or the Byzantine scale. It is a physically reactivating scale and enhances many aspects related to intelligence, such as attention, concentration, and memorization, along with the ability to make connections. This is achieved by its coherent and symmetrical interval structure, as we explained previously about Karaharapriya.

In this scale, the sequence of intervals has the same progression for both ascending and descending modes:

$$
1 / 2 \quad 1 \frac{1}{2} \quad 1 / 2 \quad 1 \quad 1 / 2 \quad 1 \frac{1}{2} \quad 1 / 2
$$

Moreover, the formula of the first part $\left(\begin{array}{lll}1 / 2 & 11 / 2 & 1 / 2\end{array}\right)$ is repeated in the second (symmetry or specularity). Finally, the presence of two long intervals ( 1 tone and $1 / 2$ ) should be noted, which implies a more complex calculation for the brain.

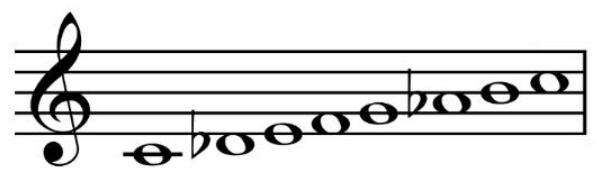

Figure 5 Double harmonic scale notation

Many other scales, cataloged in the Melakartha System, can also be used for specific problems and pathologies. Their effect is based on the emotions they activate and transform. In the singing practice of musical scales, the transformative and cathartic effect occurs in the ascending phase, while in the descending mode, the scale consolidates and reinforces the positive effects. It is for 
this reason that Pythagoras made his disciples sing only in the descending phase as they had already proceeded with clearing their emotional blocks and had to consolidate the results and obtain further skills.

\subsection{Listening to the Raga [14]}

In addition to the active practice of singing therapeutic musical scales, when the subject is unable to use his/her voice, he/she can listen to particular compositions of Indian classical music called Ragas. Each Raga is based on a precise musical scale. It is further elaborated according to a pre-established sequence that includes a long free introduction called Alaap, which is without rhythm. In Alaap, the typical mood of the composition is introduced by reposing on the main notes (vadi and samvadi). After that, the Raga develops with a theme (Gat) based on a rhythmic cycle (Tala), which is interspersed with improvisations that respect the fixed scale and the rhythmic cycle, with a crescendo in high tempo leading to the end (Jhala). Listening to a Raga for a significant duration of at least 20 to 30 minutes allows the subject to move his/her emotional energies in a significant way, with transformative effects on the energy blockages comparable to those obtained with singing. In a few hospitals in India, this procedure is well established to solve various pathologies and side effects of medicines, with detailed protocols that allow feedback from patients and members of the operating team, and the results are appreciable and verifiable. $[15,16]$

As an example, we propose the $20^{\text {th }}$ Melakartha: Nata Bhairavi (corresponding to the natural minor or the Aeolian mode), on which Raga Darbari Kanada [17] is based, which has been widely used with excellent results in the treatment of problems such as insomnia, depression, and anxiety, and as an anti-stressor during post-surgical discomfort.

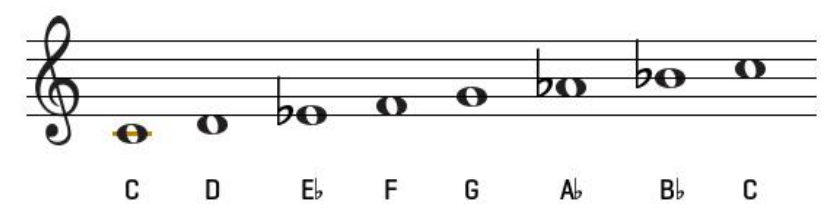

Figure 6 Aeolian mode notation — Raga Darbari Kanada

\section{Conclusions}

From the analysis of the structural and functional characteristics of therapeutic musical scales, we may conclude that we are witnessing the emergence of a great healing potential that can transform emotional energy blocks in the subjects who sing the appropriate scales by following the indicated procedure with continuity and regularity. The practice does not require long application times, and a regular daily execution lasting for about 10 minutes is sufficient. This technique (together with many others that are part of the Nāda Yoga system) is based on the ancient practices of the musical tradition of India and has always been used for psychophysical well-being. This technique has been incorporated by many traditions of the West, especially through the mediation of the Greek world. To come to the present day, it was propagated by the musician and music therapist Shri Vemu Mukunda from Bangalore in his numerous seminars and training courses in Europe in the 1990s. 


\section{Acknowledgments}

Arnab Bishnu Chowdhury for support in the translation of the original Italian text.

\section{Author Contributions}

The author did all the research work of this study.

\section{Competing Interests}

The authors have declared that no competing interests exist.

\section{References}

1. Mukherjee P. The scales of Indian music. New Delhi: Aryan Books International; 2004.

2. Daniélou A. Introduction to the study of musical scales. New Delhi: Oriental Books Reprint Corporation; 1979.

3. Porges SW. The polyvagal theory: Neurophysiological foundations of emotions, attachment, communication, and self-regulation. New York: WW Norton \& Company; 2011.

4. McCraty R, Zayas MA. Cardiac coherence, self-regulation, autonomic stability, and psychosocial well-being. Frontiers in psychology. 2014; 5: 1090.

5. Beauchaine T. Vagal tone, development, and Gray's motivational theory: Toward an integrated model of autonomic nervous system functioning in psychopathology. Dev Psychopathol. 2001; 13: 183-214.

6. From Wikipedia [CrossRef]

7. Misto di R. La Terapia musicale nei rapporti interpersonali [Internet]. Available from: http://Inx.nadayoga.it/Immagini/La_terapia_musicale.pdf.

8. Wan $\mathrm{CY}$, Schlaug G. Music making as a tool for promoting brain plasticity across the life span. Neuroscientist. 2010; 16: 566-577.

9. Gill KZ, Purves D. A biological rationale for musical scales. PLoS ONE. 2009; 4: e8144.

10. Bleyle PE. Music as a mood modulator. Retrosp Theses Diss. 1992. Doi: 10.31274/rtd-1808138092.

11. Tanaka $\mathrm{Y}$, Nogawa $\mathrm{H}$, Tanaka H. Music therapy with ethnic music for dementia patients. Int J Gerontol. 2012; 6: 247-257.

12. Bunt L, Stige B. Music therapy: An art beyond words. London: Routledge; 1994.

13. Reybrouck $M$, Vuust $P$, Brattico $E$. Music and brain plasticity: How sounds trigger neurogenerative adaptations. In Neuroplasticity Insights of Neural Reorganization. London: IntechOpen. 2018.

14. Sundar S. Traditional healing systems and modern music therapy in India. Music Ther Today. 2007; 8: 397-407.

15. Chowdhury A.B. et al. Project gratitude: An integral approach to music therapy and women's health at Shree Krishna Hospital, Gujarat, India. In Sri Aurobindo Ashram Annual Report of Research Activities 2018-19. New Delhi: Sri Aurobindo Ashram Press; 2019. 
16. Giordano F, Scarlata E, Baroni M, Gentile E, Puntillo F, Brienza N, et al. Receptive music therapy to reduce stress and improve wellbeing in Italian clinical staff involved in COVID-19 pandemic: A preliminary study. Arts Psychother. 2020; 70: 101688.

17. Mukherjee SC, Mukherjee R. Role of music therapy intervention using Raga Darbari Kanada of Indian classical music in the management of depression, anxiety, stress, and insomnia among elderly adults. Res Rev J. 2019; 4: 2008-2017.

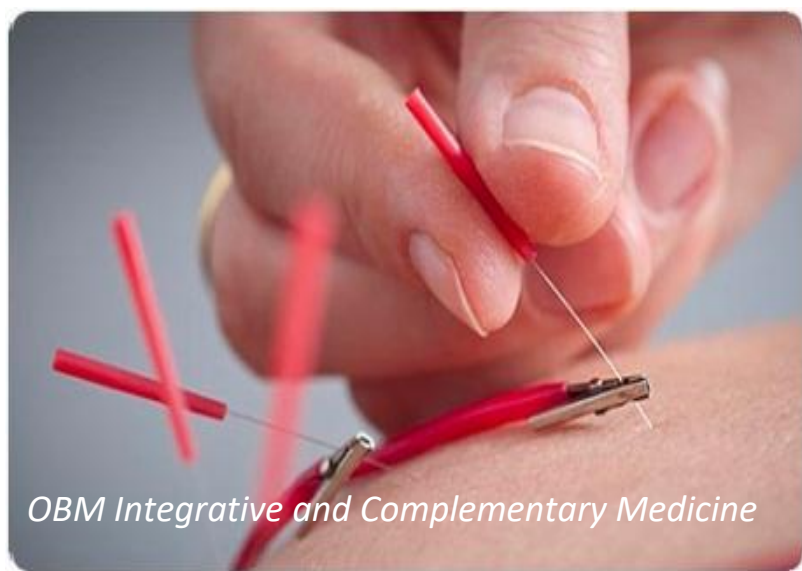

Enjoy OBM Integrative and Complementary Medicine by:

1. Submitting a manuscript

2. Joining in volunteer reviewer bank

3. Joining Editorial Board

4. Guest editing a special issue

For more details, please visit:

http://www.lidsen.com/journals/icm 